\title{
Tecnura
}

\section{Marco de referencia para la integración de recursos web como servicios de e-learning en .LRN}

\section{Reference framework for integrating web resources as e-learning services in .LRN}

\author{
Fabinton Sotelo Gómez ${ }^{1}$, Armando Ordóñez², Mario Fernando Solarte ${ }^{3}$
}

Fecha de recepción: 31 de agosto de 2014

Fecha de aceptación: 24 de agosto de 2015

Como citar: Sotelo Gómez, F., Ordóñez, A., \& Solarte, M. F. (2015). Marco de referencia para la integración de recursos Web como servicios de e-learning en .LRN. Revista Tecnura, 19(46), 79-91. doi:10.14483/udistrital.jour. tecnura.2015.4.a06

\section{Resumen}

Las plataformas de gestión de aprendizaje (LMS) como Dot LRN (.LRN) han tenido una gran difusión y uso como herramienta pedagógica. Sin embargo, a pesar de sus grandes potencialidades, la mayoría de estas plataformas no permiten integrar de forma fácil los servicios comunes en la Web. La reutilización de recursos externos en los LMS es fundamental para extender cantidad y calidad de los servicios educativos de los LMS. El presente artículo presenta un conjunto de criterios y lineamientos arquitectónicos para la integración de recursos Web para e-learning en la plataforma .LRN. Para ello se realizan tres fases: primero; se caracterizan las posibles tecnologías de integración para ser usada, segundo; se analizan los recursos Web que prestan servicios educativos y pueden ser integrados a las plataformas LMS, finalmente; se identifican algunos aspectos arquitectónicos de la plataforma relevantes para la integración. Los principales aportes de este artículo son: una caracterización de recursos Web y los servicios educativos disponibles hoy en día en la Web; y la definición de unos criterios y lineamientos para la integración de recursos Web a .LRN.
Palabras clave: e-learning, sistema de gestión de aprendizaje, lineamientos, framework

\begin{abstract}
The learning management platforms (LMS) as Dot LRN (.LRN) have been widely disseminated and used as a teaching tool. However, despite its great potential, most of these platforms do not allow easy integration of common services on the Web. Integration of external resources in LMS is critical to extend the quantity and quality of educational services LMS. This article presents a set of criteria and architectural guidelines for the integration of Web resources for e-learning in the LRN platform. To this end, three steps are performed: first; the possible integration technologies to be used are described, second; the Web resources that provide educational services and can be integrated into LMS platforms are analyzed, finally; some architectural aspects of the relevant platform are identified for integration. The main contributions of this paper are: a characterization of Web resources and educational services available today on the Web; and the definition
\end{abstract}

Ingeniero de Sistemas, magister en Ingeniería Telemática. Docente de la Fundación Universitaria de Popayán. Popayán, Colombia. Contacto: fabinton.sotelo@unicauca.edu.co

2 Ingeniero Electrónico, doctor en Ingeniería Telemática. Docente de la Fundación Universitaria de Popayán. Popayán, Colombia. Contacto: jaordonez@unicauca.edu.co

3 Magíster en Ingeniería Área Telemática, estudiante de Doctorado de Ingenieria Telemática. Profesor de planta de la Universidad del Cauca. Popayán, Colombia. Contacto: msolarte@unicauca.edu.co 
of criteria and guidelines for the integration of Web resources to .LRN
Keywords: e-learning learning management system, guidelines, framework.

\section{INTRODUCCIÓN}

El auge de las tecnologías de la información y la comunicación (TIC) en el campo educativo ha llevado al surgimiento y posicionamiento de la educación electrónica, o E-learning (Cabero, 2006) soportada por las plataformas de gestión de aprendizaje (Learning Management Systems, LMS). Un LMS es una herramienta que realiza, entre otras funciones: la mediación de la apropiación de conocimientos, la administración de los procesos de enseñanza, el acceso a herramientas didácticas y de comunicación. Con estas plataformas, las instituciones educativas dan acceso a cursos presenciales, semipresenciales y a distancia, y ofrecen el servicio educativo a mayor número de estudiantes al facilitar el acceso a quienes tienen dificultad de traslado a las clases presenciales (Boneu, 2007). En nuestro entorno, algunas Universidades dan soporte a cursos de pregrado y posgrado a través de diferentes tecnologías: la Universidad del Cauca, por ejemplo, utiliza ".LRN", y la Fundación Universitaria de Popayán utiliza Moodle.

Dos de los LMS open source más utilizados son Moodle y dotLRN. Moodle es un paquete de software libre basado en principios pedagógicos que permite la construcción de comunidades de aprendizaje en línea. DotLRN es una plataforma "empresarial" de software libre para soportar e-learning y comunidades virtuales. Aunque la elección de una plataforma corresponde exclusivamente a los requerimientos de cada institución o proyecto, DotLRN ofrece un gran conjunto de ventajas debido a su enfoque empresarial (Martin, 2007).

La integración de recursos web dentro de los LMS permite la reutilización de contenidos disponible en internet y potencia la cantidad y calidad de los servicios educativos que pueden prestar los LMS. Igualmente, la integración de recursos web evita situaciones de distracción en el estudiantado al consultar recursos educativos en la web. Si los recursos web están integrados al LMS, se evita que el estudiante deba salir de la plataforma en el desarrollo del proceso formativo, por lo que este aspecto es indirectamente una forma de mantenerlo concentrado con un mejor aprovechamiento del tiempo. Numerosos trabajos acerca de la integración de recursos web como servicios educativos se han realizado utilizando la plataforma Moodle; sin embargo, son escasos o muy puntuales los trabajos que se han centrado en la integración de dichos recursos utilizando .LRN. Esta integración plantea grandes retos debido a la arquitectura y el lenguaje de programación tan particulares (Sotelo \& Solarte, 2013).

Para el contexto de este trabajo, se definen los siguientes conceptos:

- Marco de referencia: articulación de tres elementos: 1. marco conceptual; 2. lineamientos de índole arquitectónica y 3 . verificación de los lineamientos con el desarrollo de un caso de estudio.

- Recurso web: los elementos identificados por una URI (identificador uniforme de recurso) alojada en Internet y accedida mediante una versión del protocolo HTTP, según el ISBD ER (Estándar International de Descripción Bibliográfica) y la W3C (consorcio de la red alrededor del mundo).

- Servicio de e-learning: se basan en computación en la nube (por ejemplo Google Docs o Google Forms, u otros de la web 2.0 como plataforma de acciones educativas); para esto se toma la definición de servicio contexto propuesto por De la Fuente (2011) y W3C (1999).

En el presente artículo se describe un marco de referencia basado en criterios de integración y lineamientos arquitectónicos para integrar recursos web que presten servicios educativos en .LRN, y se organiza de la siguiente manera: en la sección 2 
se presentan los conceptos que enmarcan este trabajo; en la 3 se presenta la caracterización de los recursos web externos al LMS; en el 4, se ofrece la definición de criterios para integrar recurso; en el 5, los lineamientos arquitectónicos; y, por último, en el 6, las conclusiones.

\section{SISTEMA DE GESTIÓN DE APRENDIZAJE .LRN}

Un LMS proveen entornos virtuales de aprendizaje (EVA) que incorporan herramientas necesarias para que los docentes faciliten un proceso de aprendizaje en los estudiantes. Los LMS buscan simular de la mejor forma un entorno real (Zapata, 2003; Matesanz \& López, 2009).

El software de código abierto .LRN ha sido creado usando el framework de aplicaciones web OpenACS; así los usuarios de .LRN se nutren de servicios desarrollados para .LRN, y otros desarroIlos para OpenACS (.LRN, 2005, 2010). OpenACS es utilizado para la construcción de aplicaciones y sitios web orientada a comunidades de forma modular, donde su estructura se basa en paquetes, permite la gestión de usuarios, grupos, contenidos, foros, búsqueda de textos y gestión de actividades educativas (en la cual se enmarca .LRN); cuenta con un servidor web OALserver que cumple las funciones de servidor de aplicaciones, es libre, multihilo y escalable.

Al contrario de otros LMS que se enfocan en la gestión de contenidos de docentes y estudiantes, .LRN se centra en facilitar la comunicación entre los actores de la experiencia de aprendizaje. Así, desde el registro se da la opción al estudiante de compartir documentos, contar con una administración de usuarios y gestión de comunidades. OpenACS cuenta con herramientas para desarrollo en su lenguaje de programación nativo llamado TCL (tWSDL Project, 2010). Además, soporta varios motores de bases de datos tales como Oracle y PosgreSQL.

La estructura modular de OpenACS permite personalizar y crear nuevas aplicaciones de forma rápida; el espacio de usuario es organizado a través de portlets, cada uno de de los cuales da acceso a sus servicios disponibles. Se destaca que

\begin{tabular}{|c|c|c|}
\hline \multicolumn{3}{|c|}{ 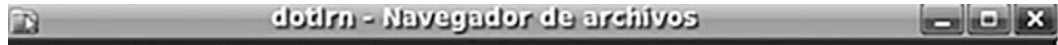 } \\
\hline \multicolumn{3}{|c|}{ Archivo Editar Ver Ir Marcadores Ayuda } \\
\hline$\circlearrowright$ Atrás $\nabla \Rightarrow$ Adelante $\vee \hat{0}$ & (x) & Q $50 \%$ Q $\nabla$ \\
\hline (2) usr share dotirn & & \\
\hline Nombre & Tamaño & Tipo \\
\hline$+\square$ apm-workspace & 1 elemento & Carpeta \\
\hline$+{ }^{b i n}$ & 13 elementos & Carpeta \\
\hline 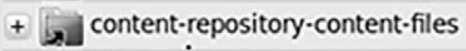 & 3 elementos & Enlace hacia Carpeta \\
\hline$+G_{\Delta}$ etc & 8 elementos & Enlace hacia Carpeta \\
\hline+[]$_{\infty} \log$ & 23 elementos & Enlace hacia Carpeta \\
\hline$+\square$ packages & 95 elementos & Carpeta \\
\hline$+\mathrm{tcl}_{\mathrm{tc}}$ & 2 elementos & Carpeta \\
\hline$+[$ www & 16 elementos & Carpeta \\
\hline 를 ChangeLog & $7,3 \mathrm{KiB}$ & Documento de registro de cambi \\
\hline 5. install.xml & $4,1 \mathrm{KiB}$ & Documento XML \\
\hline E readme.txt & $1,9 \mathrm{KiB}$ & Documento de texto sencillo \\
\hline
\end{tabular}

Figura 1. Directorios .LRN

Fuente: Elaboración propia. 
la plataforma OpenACS ofrece varias herramientas para interactuar con agentes externos a su propio núcleo de módulos, con lo que queda abierta la posibilidad de interacción con otros LMS y recursos web externos a ella.

Debido a la estructura modular de dotLRN, un análisis de los directorios permite tener una visión clara de su funcionamiento. La figura 1 presenta el conjunto de directorios que se crean al momento de la instalación de dotLRN.

Descripción de los directorios:

bin: se almacenan archivos ejecutables y scripts para el mantenimiento del servidor.

content-repository-content-file: incluye los elementos del repositorio de contenidos.

etc: almacena scripts de instalación y archivos de configuración de la plataforma.

packages: se almacenan directorios de servicios y aplicaciones del LMS

log: almacena errores y logs de acceso del servidor.

tcl: almacena datos y llamadas de inicio al servidor web de OpenACS.

$w w w$ : almacena páginas que no se encuentran en los paquetes, como contenido estático y páginas personalizadas.

Este trabajo se concentra en packages, puesto que en él se almacenan los paquetes que se desarrollan. En su estructura se encuentra: el modelo de datos, las librerías, la lógica de programación, las páginas de administración, las páginas de usuario de un paquete.

.LRN presenta los siguientes servicios a los usuarios en el entorno virtual de aprendizaje (San Cristóbal, 2010): calendario, blog, documentos, wiki, creación y gestión de comunidades, repositorios de contenido, cuestionarios y evaluación, foros (Universidad de Valencia, s. f.), lista de miembros, FAQs, internacionalización i18n, autenticación (MIT, 2013, IETF, 2013), tareas, seguimiento de usuarios, sistema de repositorio de objetos de aprendizaje - LORS, álbum de fotos, WebDAV, ECommerce, WysiWyg - editor Web, WimpyPoint, AJAX (Eguiluz, 2007), Templating.

\section{TRABAJOS RELACIONADOS CON LA INTEGRACIÓN DE RECURSOS WEB EXTERNOS A LMS}

En este apartado se relacionan investigaciones donde se han integrado servicios externos y se identifican otros servicios con los que no cuenta .LRN, para hacer una caracterización de ellos.

\section{Trabajos relacionados}

Garita (2008) presenta una integración de la plataforma de servicios internos del Instituto Tecnológico de Costa Rica con .LRN para crear nuevos servicios a estudiantes, docentes y administrativos; estos servicios están orientados al Departamento de Admisión y Registro, y usa un framework de .NET, consumidos en .LRN, instalando TcISOAP y el tsoap.

Raffenne (2008) presenta la integración de .LRN con un framework de arquitectura de servicios abierta basada en SOA para la adaptación de servicios y personalización de contenidos; las interfaces son implementadas usando servicios web, W3C SOAP y REST.

Martin (2007) diseña dos middleware capaces de dar respuestas de forma rápida y automática a preguntas que son comunes en comunidades educativas virtuales; este sistema recibe las solicitudes de desde diferentes plataformas que pueden ser Moodle, .LRN o correo electrónico y buscar respuesta en un repositorio conocimiento que puede incluir LMS, Google o bases de datos institucionales, esta plataforma soporta diversos estándares entre los que se encuentra SCORM.

Delgado (2010) realiza un estudio detallado de los estándares y especificaciones existentes como IEEE, proyecto OKI, IMS, ADL y AICC a nivel de datos y servicios e-learning, e identifica falencias en aspectos de reutilización e interoperabilidad entre plataformas a la hora de compartir contenidos y servicios. Por esta razón, se proponen especificaciones de datos para cada servicio de los LMS, una especificación de modelo de alto nivel 
de cursos en un LMS. Como producto se desarrolla una herramienta de edición para las nuevas especificaciones propuestas de servicios educacionales de los LMS generando archivos ICOPER (Sunil, 2009, Icoper, 2011) busca detectar y resolver problemas de interoperabilidad, ayudando a completar las herramientas disponibles para garantizar el intercambio de material de evaluación entre algunos LMS (Moodle. LRN y CLIX).

Haciendo uso del repositorio de ICOPER "open ICOPER Content Space (OICS)", Gutiérrez (2010) desarrolla un prototipo para administrar y compartir recursos de evaluación de forma fácil con el repositorio, haciendo uso del modelo de referencia ICOPER y mediante una capa definida como "Middle Layer API". En el caso de uso se demuestra el acceso desde .LRN al repositorio. Similarmente, Agea (2010) se desarrolla un prototipo para el acceso de material de e-learning y evaluación desde OICS a .LRN bajo el estándar IMS-QTI.

Rivera \& Chingal (2011) investigaron a nivel técnico la interoperabilidad de servicios entre las plataformas Moodle y .LRN, mediante procesos y servicios web. La arquitectura planteada se basa en una arquitectura orientada a servicios (SOA). En el modelo arquitectónico, los dos LMS implementan servicios web basados en SOAP (Simple Object Access Protocol), publicados por medio de WSDL, y se dispone de un servidor de WS-BPEL que ejecuta procesos a partir de los servicios web dispuestos. Se implementa con Moodle2, ya que presenta características para orquestación de servicios. Según el estudio de caso desarrollado, se concluye que los servicios web disponibles en Moodle y .LRN no son compatibles para el manejo de BPEL. Con la implementación de un intermediario/adaptador se brinda compatibilidad de los servicios web de las plataformas y al mismo tiempo se adecua la información; sin embargo, la interoperabilidad alcanzada por este medio no es suficientemente satisfactoria.

Por su parte, De la Fuente (2011) investiga servicios externos a plataformas de aprendizaje para ser incorporados a través del framework GSI (servicio genérico de integración), como un complemento al estándar IMS-LD implementado como una extensión del GRAIL (Gradient-lab RTE for Adaptive IMS LD in .LRN) que permite el intercambio bidireccional de información entre IMS LD implementado en una plataforma de e-learning (se utilizó .LRN) y las herramientas de terceros. Como caso de estudio se realiza una integración de Google Forms (Google, 2011) y Google Spreadsheets (Google, 2011) a un proceso de aprendizaje para usarlo como evaluación.

La arquitectura planteada en esta investigación se enfoca y trabaja solo en el modelo del diseño del curso IMS-LD.

A su vez, Xu (2003) presenta un framework entre dos LMS capaz de hacer interactuar contenidos y servicios de aprendizaje mediante el uso de una arquitectura orientada a servicios; si bien este trabajo aporta una conceptualización de su arquitectura, es aplicable a LMS capaces de exponer y consumir sus recurso mediante servicios web, el cual no sería el caso de .LRN.

Finalmente, Dagger (2007) presenta una evolución tecnológica respecto a la prestación de servicios de e-learning, desde sus principios, cuando se resaltaba la importancia de la interoperabilidad de contenidos, hasta las nuevas generaciones, donde se destaca el compartir herramientas, la semántica, la flexibilidad y las plataformas en contextos orientados a servicios, soportados en estándares SOA y WDSL, entre otros. El contexto de este trabajo se enfoca solo en el consumo de servicios e-learning, expuesto como servicios web, y no a cualquier recurso educativo expuesto en la web, que sí es el propósito de nuestra investigación.

\section{Caracterización de recursos web externos}

En esta sección se menciona y caracteriza recursos orientados a prestar servicios educativos que no se encuentran en la plataforma y son de utilidad a los procesos de aprendizaje en un contexto educativo definido. Estos están disponibles en la web y son accedidos haciendo sólo uso del protocolo HTTP 


\section{Traductores de idiomas online}

La necesidad inmediata de traducir al idioma nativo del estudiante lleva a pensar en la facilidad de incluir un traductor de palabra o texto en la plataforma, entre estos se destacan los siguientes recursos: Babylon, (traductor.babylon.com/); Google traductor (translate.google.es/); El mundo.es (www. elmundo.es/traductor/).

\section{Mapas conceptuales}

Estas herramientas permiten el trabajo colaborativo en línea, en esta categoría tenemos: Cmaptools (Arias, 2009); bubbl.us (https://bubbl.us/); wisemapping (http://www.wisemapping.com/c/home. htm); origramy (http://my.origramy.com); mindmeister (http://www.mindmeister.com); Kmap (http://ka-map.maptools.org/\#).

\section{Enciclopedias online}

Recursos para la consulta académica: Kalipedia (http://www.kalipedia.com/); Wikipedia (http:// es.wikipedia.org); Enciclopedia Libre Universal en Español (http://enciclopedia.us.es); Wikilengua (http://www.wikilengua.org).

\section{Mapas online}

Para apoyar cursos de geografía, historia, sociales, biología, entre otros: Google Maps (http://maps.google. es/); Mapred (http://www.mapred.com/es); Mapas. co (http://beta.mapas.com.co/); Instituto Geográfico Agustín Codazzi (http://geoportal.igac.gov.co).

\section{Redes sociales}

La redes sociales en la actualidad son una de las aplicaciones de la web 2.0 con mayor utilización en Internet; su aplicación educativa se da en la discusión de temas específicos, trabajos colaborativos y forma de comunicación. Por mencionar algunos ejemplos tenemos: http://facebook.com, Google+: https://plus. google.com, Linkedin: https://co.linkedin.com/.

\section{Simuladores y juegos interactivos}

Estas herramientas son de importante utilidad en los procesos de aprendizaje; se han identificado sitios especializados en esta área, tales como: recursos de la Estación Espacial Internacional-ISS (http://esamultimedia.esa.int/docs/issedukit/es/ html/index.html); entrenar el cerebro (http://i.lumosity.com). Recursos para diferentes áreas del conocimiento (http://phet.colorado.edu/es/simulations/category/new). Recursos del Ministerio de Educación de Colombia: ICFES interactivo (http:// www.icfesinteractivo.gov.co/); Colombia aprende (http://www.colombiaaprende.edu.co).

\section{METODOLOGÍA}

Esta sección presenta la metodología propuesta para la integración de recursos web en dotLRN.

\section{Definición de criterios para integración de recursos web como servicios de e-learning en .LRN}

En este apartado se estudia las tecnologías usadas y se definen los criterios para realizar una óptima integración de recursos web a .LRN

Antecedentes en integración de recursos web La integración se define como la combinación de diferentes elementos software con el objeto de formar o completar un sistema y dar un valor agregado (Abad, 2013)..LRN, es una plataforma de código libre, diseñada por módulos y que soporta el intercambio de datos a través de las siguientes tecnologías: HTTP y sus métodos: estos métodos de petición usados por el protocolo de transferencia de hipertexto (HTTP) se definen en la RFC 2616 (Network Working Group, 2000). WS (Web Services) (W3C, 2010): Permiten la comunicación de componentes software desarrollados en diferentes lenguajes de programación se ejecuten en diferentes sistemas operativos (Corrales, 2008). Son parte fundamental de la arquitectura orientada a servicio SOA (Service Oriented Architecture), la cual define la interoperabilidad de servicios para soportar requisitos software del usuario (Besemer, 2008). Algunas tecnologías asociadas con los servicios Web 
son: WSDL (Web Services Description Language): es un formato $X M L$ que se usa para describir servicios web (W3C, 2001). SOAP (Simple Object Access Protocol): Permite intercambiar información $\mathrm{XML}$ en contextos no centralizados; soporta protocolos como HTTP y SMTP (W3C, 2007). REST (Representational State Transfer): describe interfaces de los servicios web utilizando los métodos de HTTP. Los sistemas bajo este paradigma son Ilamados RESTful (Fielding, 2000). BPEL (Business Process Execution Language): es un estándar XML para la orquestación de servicios. (OASIS, 2003).

\section{Requisitos del servicio a integrar}

La caracterización de recursos permite establecer que la mayoría de estos se acceden usando el protocolo HTTP, el cual posibilita el envío y recepción de datos entre la plataforma y el servidor que aloja el recurso web a integrar, permitiendo trabajar con diferentes funcionalidades como Flash, Java, Javascript, HTML, HTML5, web 2.0, etc. Debido a que en la web se pueden encontrar muchos recursos HTTP, es necesario establecer requisitos mínimos que garanticen su integración con .LRN, los cuales se describen a continuación:

1. Es indispensable que posea una URL. Ejemplo: http://phet.colorado.edu/sims/ohms-law/ohmslaw_es.html. No es elegible para integrar un servicio e-learning que no tenga una URL. Ejemplo: Stellarium (software capaz de mostrar un cielo realista en 3D). Se instala de manera local y no se expone por URL.

2. No se debe integrar todo el sitio web al que pertenece el servicio, se debe identificar la URL del recurso.

Ejemplo incorrecto: http://www.colorado.edu/. Esta URL accede al sitio web de la universidad donde se encuentra el recurso deseado, pero no identifica el recurso.

Ejemplo correcto: http://phet.colorado.edu/ sims/ohms-law/ohms-law_es.html. Esta URL pertenece al sitio web anterior e identifica exactamente al recurso deseado.
3. Se debe tener en cuenta el tamaño de visualización del recurso, ya que será integrado dentro del sitio web de .LRN, por lo que tendrá menor espacio de lo usual. El tamaño máximo depende de factores como la resolución promedio de las pantallas clientes de la plataforma, por lo que se recomienda programar el recurso en un tamaño de $20 \mathrm{~cm}$ de ancho por $14 \mathrm{~cm}$ de alto.

4. Para las funcionalidades compatibles con HTTP (como Flash, Java, Javascript, HTML, HTML5, web 2.0, etc.) se deben cumplir los anteriores requisitos. Ejemplo: Google Maps presenta funcionalidades javascript que deben ser programadas y expuestas en una URL.

5. En cuanto a los navegadores web (Internet Explorer, Mozilla Firefox, Opera, Google Chrome, Safari, entre otros) deben contar con los plugins necesarios para ejecutar los recursos web que consume mediante la plataforma.

6. El recurso web a integrar debe prestar un servicio e-learning en la plataforma que apoye un proceso de aprendizaje; ejemplo: Google Maps por sí solo no es un servicio e-learning, pero para el contexto del proyecto puede ser usado por los docentes del área de ciencias sociales para enseñar geografía.

Los anteriores criterios para integrar los recursos deben ser seguidos por el docente (y tenidos en cuenta por el administrador) en el momento que desee integrar un servicio educativo a un curso a su cargo; con esto garantiza al administrador del LMS una óptima integración a la hora de desarrollar los paquetes necesarios para dicha integración.

\section{RESULTADOS}

\section{Lineamientos arquitectónicos para la integración de recursos web en .LRN}

En este punto se establecen los lineamientos arquitectónicos para integrar recursos web que presten servicios e-learning en el LMS .LRN teniendo en cuenta su arquitectura. 


\section{Estructura de una aplicación en .LRN}

En el desarrollo de un curso, un docente ve la necesidad de incluirle servicios que no están presentes; la manera que .LRN soluciona esto es por medio de applets, portlets y paquetes. Por esta razón el camino para integrar recursos web a .LRN es desarrollando aplicaciones basadas en estos tres elementos:

1. Paquete: contiene tanto el modelo de datos como la lógica y funcionamiento del paquete. Así como la interfaz de usuario del paquete; para el caso de integración, es aquí donde se hace la programación para referenciar la URL del recurso web.

2. Portlet: proporciona la interfaz de usuario para los portales de la plataforma; aquí se programa la interfaz grafica del paquete y su administración describiendo el recurso web y vinculándolo al enlace del paquete.

3. Applet: utiliza la interfaz de los portlets y establece las propiedades para el portal de .LRN, haciendo posible que la aplicación sea agregada por los administradores o docentes del curso.

Ejemplo: en la figura 2 se visualizan los tres directorios que se crean en una aplicación que integra un recurso web Ilamado Geografía6 a .LRN; sus rutas son:

Para el Paquete: /usr/share/dotlrn/packages/ geografia6

Para el Portlet: /usr/share/dotlrn/packages/ geografia6-portlet

Para el Applet: /usr/share/dotlrn/packages/ dotlrn-geografia6

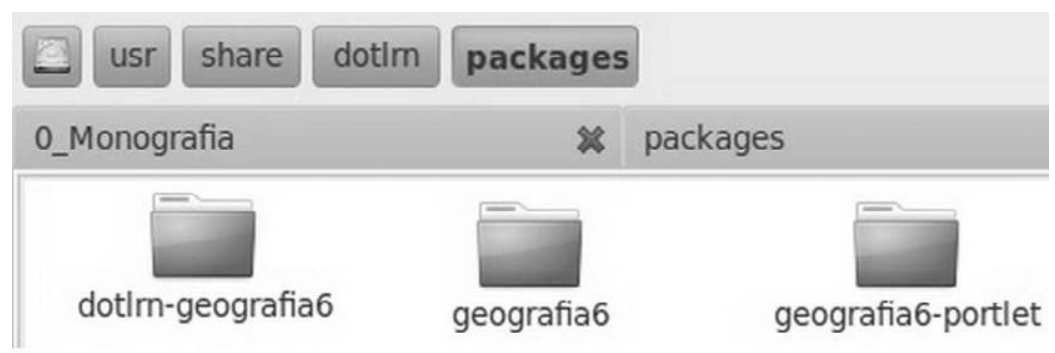

Figura 2. Directorios de aplicación .LRN.

Fuente: Elaboración propia

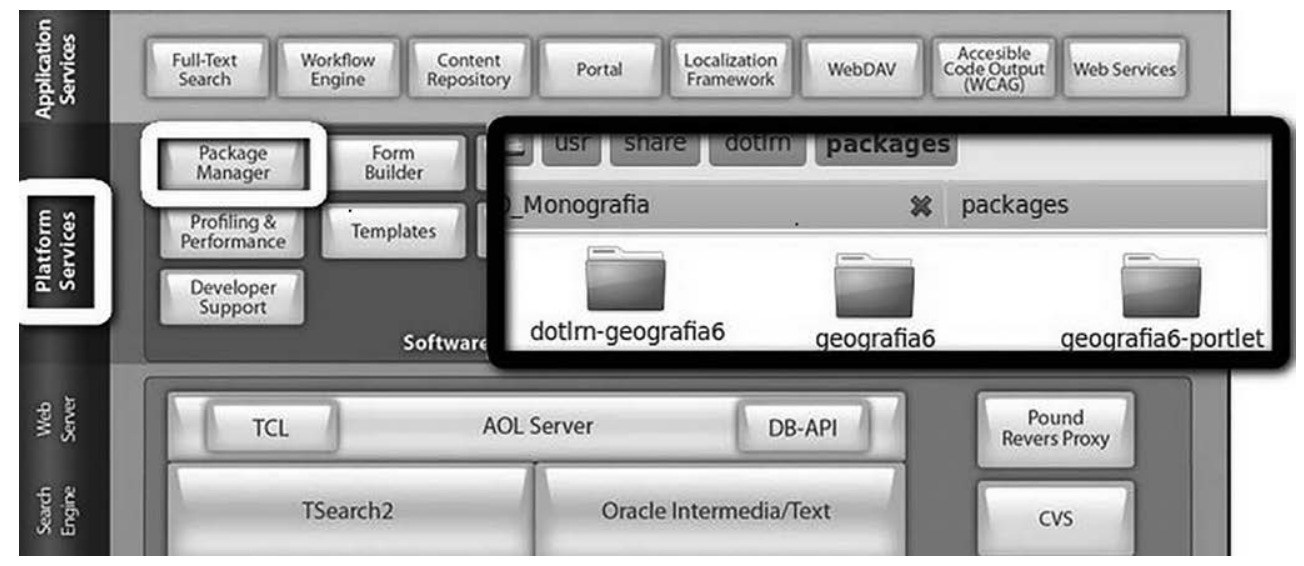

Figura 3. Aplicación en. LRN.

Fuente: Elaboración propia. 


\section{Desarrollo de una aplicación que integra recursos web a .LRN}

Se debe tener en cuenta que el desarrollo de la aplicación a nivel arquitectónico de .LRN se encuentra en la capa de paquetes y es desarrollado por un usuario con privilegios de administrador (Figura 3).

A continuación se establecen los lineamientos para la integración de recursos web que presten servicios e-learning en .LRN mediante la creación de una aplicación basada en paquetes, portlets y applets que directamente se integra en la arquitectura del LMS en la capa de la plataforma de servicios:

1. Crear el paquete por medio del Package Manager (http://localhost:9000/acs-admin/apm/). Una vez creado el paquete, este se encuentra vacío, instalado y listo para ser programado (/usr/share/ dotlrn/packages/Nombre_Paquete).

2. Programar el paquete para hacer referencia al recurso web a integrar (/usr/share/dotlrn/packages/ Nombre_Paquete/www/index.apd), teniendo en cuenta los criterios de integración de recursos web de la sección anterior.

3. Crear los directorios del portlet y el applet. Esta tarea se hace de forma automática haciendo uso del script de Nima Mazloumi disponible en los foros de OpenAcs (http://openacs.org/forums/ message-view?message_id=299982); estos se deben copiar en el directorio de paquetes para luego en su momento ser instalados.

4. Programar el portlet para describir el recurso web y vincularlo al paquete, (/usr/share/dotIrn/packages/Nombre_Paquete-portlet/www/ Nombre_Paquete-portlet.adp) y desarrollar su administración (/usr/share/dotlrn/packages/Nombre_Paquete-portlet/www/Nombre_Paquete-admin-portlet.adp).

5. Programar el applet solo si es necesario (/usr/share/dotlrn/packages/dotlrn- Nombre_Paquete /tcl/ dotIrn-Nombre_Paquete-procs.tcl).

6. Instalar el applet y el portlet por medio de la herramienta de instalación de software de .LRN en la opción Install from Local (http://localhost:9000/ acs-admin/install/).

7. Finalmente la aplicación está disponible para ser agregada en un curso desde la opción Administrar applets en el portal Admin del curso por el docente encargado.

Estos lineamientos deben ser seguidos por el administrador del LMS o el desarrollador a cargo de integrar los servicios a la plataforma.

En las figuras 4 a 7 se visualizan los pasos para agregar un recurso web en un curso, para el ejemplo, Google Maps.

\section{CONCLUSIONES Y TRABAJO FUTURO}

En el marco de referencia propuesto se define una caracterización de los recursos web externos que presten servicios e-learning a .LRN, la cual permite tener claridad de las tecnologías usadas y aspectos para integrar; así mismo se definen criterios de integración y se plantean lineamientos de índole arquitectónica para integrar recursos web en la plataforma, siguiendo su patrón de arquitectura Modelo Vista Controlador (MVC).

Con el marco de referencia para la integración de servicios externos a .LRN se da la posibilidad de usar más y mejores recursos en la plataforma y apoyar procesos educativos según el contexto en que se use. Con este marco se permitirá la reutilización de servicios externos a la plataforma, saliéndose de un contexto definido tecnológicamente por estándares e investigaciones enfocadas en la reutilización de contenidos.

En experimentación realizada en un caso de estudio con dos cursos (Geografía y Física) en un colegio de zona rural del departamento del Cauca (Colombia), se pudo ratificar que los criterios de integración de recursos web en la plataforma resultan claros y de fácil entendimiento a la hora de escogerlos y que los lineamientos arquitectónicos planteados tienen resultados exitosos en el contexto de laboratorio y caso de estudio en el momento de puesta en marcha de la plataforma. 


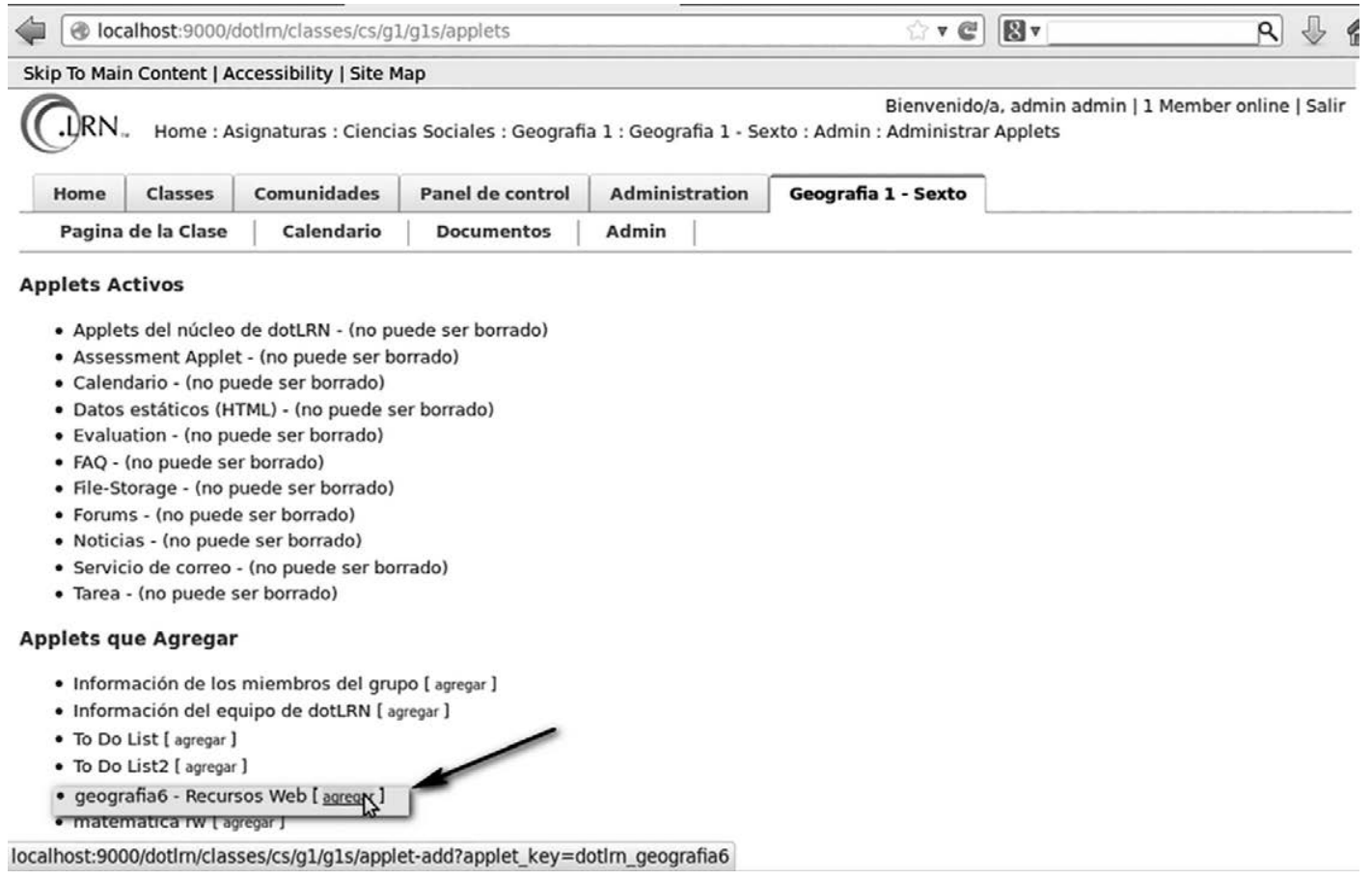

Figura 4. Applet de recurso web antes de ser agregado

Fuente: Elaboración propia.

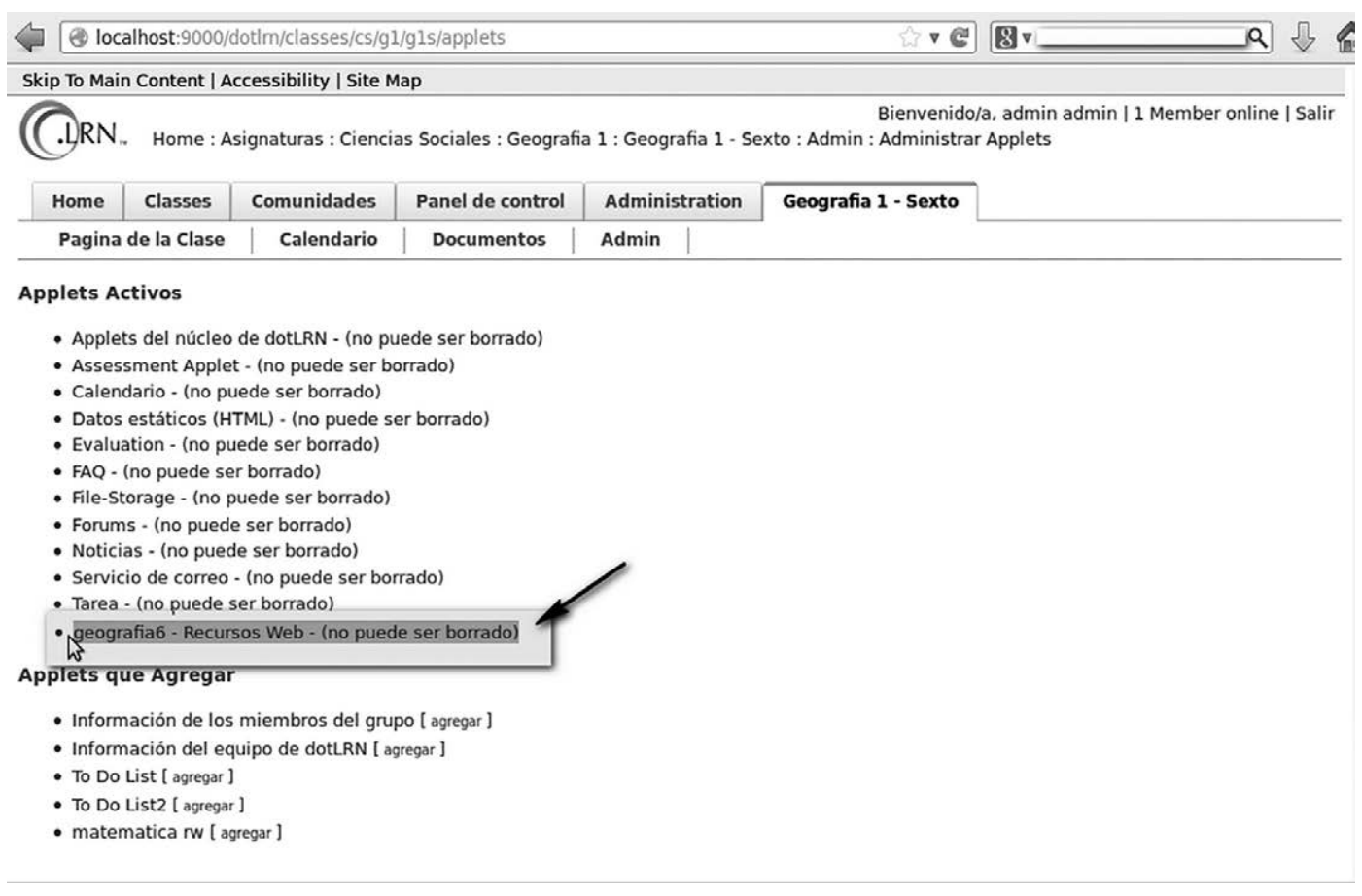

Figura 5. Applet de recurso web agregado.

Fuente: Elaboración propia. 


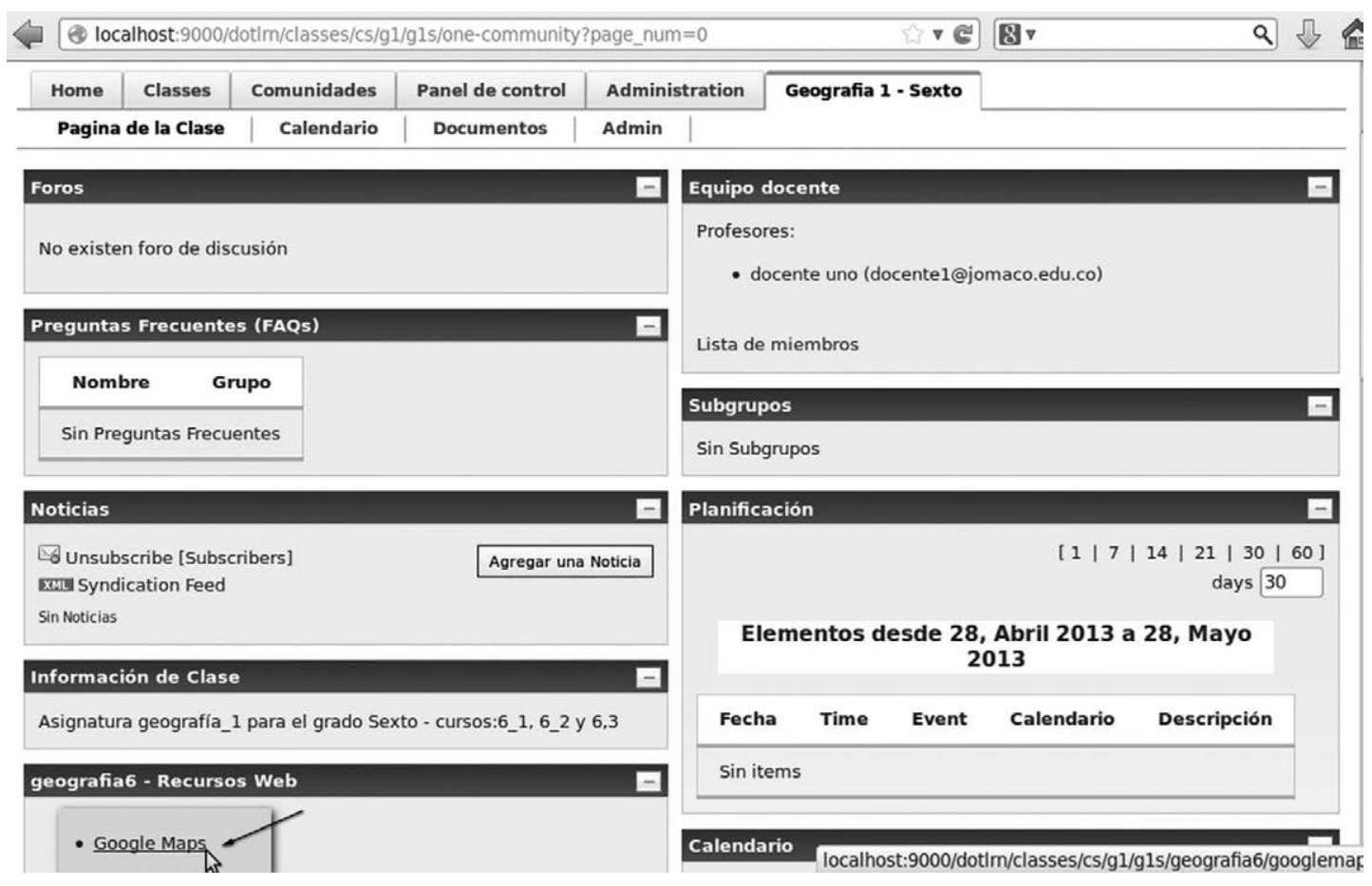

Figura 6. Portlet de recurso web integrado en un curso

Fuente: Elaboración propia.

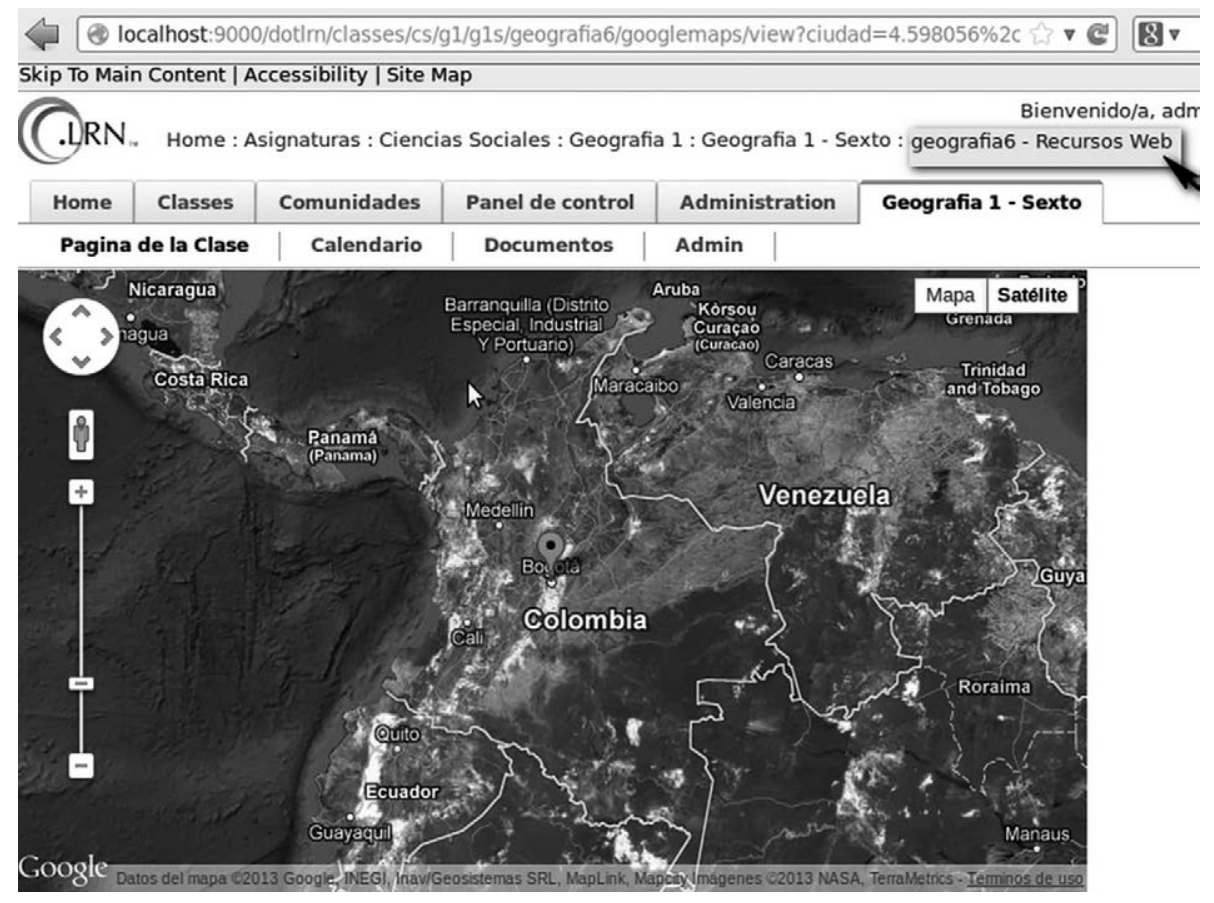

Figura 7. Vista de recurso web GoogleMaps integrado en .LRN.

Fuente: Elaboración propia. 
En este caso, los docentes afirmaron que se evidenciaba un mejor rendimiento de los estudiantes que hicieron uso de la plataforma respecto a los que solo recibieron la clase de modo convencional. Como trabajo futuro se plantea una arquitectura que integre mediante servicios web recursos o servicios propios de otro LMS, por ejemplo de Moodle.

\section{FINANCIAMIENTO}

El presente artículo se enmarca en el trabajo de maestría denominado "Marco de referencia para la integración de recursos web como servicios de E-Learning en .LRN", de la Universidad del Cauca, inscrito a su vez en el proyecto "Software Libre en Teleformación" - SOLITE, financiado por el Programa Iberoamericano de Ciencia y Tecnología para el Desarrollo (CYTED), en el área de Tecnologías de la Información y las Comunicaciones.

\section{AGRADECIMIENTOS}

Los autores agradecen a CYTED por la financiación recibida, al Grupo de Investigación en Ingeniería Telemática de la Universidad del Cauca; a los doctores Gustavo Ramírez, Álvaro Rendón y al magíster Carlos Serrano por las apreciaciones, a la Fundación Universitaria de Popayán y a la Institución Educativa José María Córdoba por permitir el estudio de caso.

\section{REFERENCIAS}

.LRN. (2005). About .LRN-Learn, Research, Network. Retrieved from http://dotlrn.org/about/

.LRN. (2010). About-Openacs. Retrieved from http:// dotlrn.org/about/openacs/

Abad, P. (2013). Estándares y Recomendaciones del OGC y CSG. Instituto Geográfico Nacional, España.

Agea, A. (2010). Production flow description and prototype for the two platforms under study (Moodle and .$L R N$ ) including the required steps to exchange the material in both platforms. ICOPER, ECP 2007 EDU.
Arias, A. (2009). Integración del CmapServer a Sistemas de Gestión de. Presentado en: Conferencia E-Learning y Código Abierto. ISBN 978-9968-641-00-5. Costa Rica.

Besemer, D. (2008). Service Oriented Architecture. (1 ed). EE.UU: Westminster Promotions.

Boneu, J. (2007). Plataformas de e-learning para el soporte de contenidos educativos abiertos. Revista de Universidad y Sociedad del Conocimiento (RUSC), 3(1).

Cabero, J. (2006). Bases pedagógicas del e-learning. Revista de Universidad y Sociedad del Conocimiento (RUSC), 3(1).

Casany, M. J., Alier, M., Mayol, E., Piguillem, J., Galanis, N., García Peñalvo, F. J., \& Conde, M. Á. (2012). Moodbile: A Framework to Integrate m-Learning Applications with the LMS.. Journal of Research and Practice in Information Technology, 44 (2), 129 -149

Corrales, J. (2008). Behavioral matchmaking for service retrieval. (Tesis doctoral, University of Versailles Saint-Quentin-en-Yvelines). Francia.

Dagger, D., O'Connor, A., Lawless, S., Walsh, E., \& Wade, V. P. (2007). Service-oriented e-learning platforms: From monolithic systems to flexible services. Internet Computing, IEEE, 11(3), 28-35.

De la fuente, L. (2011). Orchestration of learning activities through the integration of third-party services in IMS Learning Design. (Tesis doctoral, Universidad Carlos III de Madrid). España.

Delgado, C. E. (2010). Some research questions and results of UC3M in the eMadrid Excellence Network. Presentado en: EDUCON 2010 IEEE Annual Global Engineering Education Conference 1416. Madrid, España.

Delgado, C. (2005). Variación sobre XML. Novática, 173.

Eguiluz, J. (2007). Ajax. Recuperado de http://www.librosWeb.es/ajax/

Fielding, R. (2000). Architectural Styles and the Design of Network-based Software Architectures. (Tesis Doctoral, Universidad de California). EE. UU.

Garita, J. (2008). Integrating OpenACS/dotLRN with an Open and Accessible Service Architecture Framework. $8^{\text {th }}$ OpenACS/.LRN Conference TEC. Costa Rica. 
Google. (2011). Google forms. Recuperado de http:// www.google.com/google-d-s/forms/ (consultado en enero de 2012).

Google. (2011). Google Spreadsheets. Recuperado de http://www.google.com/google-d-s/spreadsheets/ (consultado en enero de 2012).

Gutiérrez, I. (2010). Management of Assessment Resorces in a Federated Repository of Educational Resource. Spinger, 6383, 139-150.

Icoper. (2011). Reference Model. Recuperado de http:// icoper.org/

IETF. (2013). The Secure Sockets Layer (SSL) Protocol Version 3.0. Recuperado de http://tools.ietf.org/ $\mathrm{html} / \mathrm{rfc} 6101$

Martin, S. (2007). Interoperability between the new open e-learning platforms: an intelligent answering machine. Universidad Nacional de Educación a Distancia: España.

Matesanz, M., \& Lopez, C. (2009). Las plataformas de aprendizaje: del mito a la realidad. Revista de innovación educativa, Univercidad de Valencia, 3, 146-148.

MIT. (2013). Kerberos: The Network Authentication Protocol. Recuperado de http://web.mit.edu/kerberos/

Network Working Group. (2000). 2616, RFC-Hypertext Transfer Protocol - HTTP/1.1. Recuperado de http://www.w3.org/Protocols/rfc2616/rfc2616.html

OASIS. (2003). OASIS Web Services Business Process Execution Language (WSBPEL) TC [estándar].

Raffenne, E. (2008). Integrating OpenACS/dotLRN with an Open and Accessible Service Architecture Framework. $8^{\text {th }}$ OpenACS/.LRN Conference TEC. Costa Rica.

Rivera, C., \& Chingal, H. (2011). Estudio de viabilidad técnica para la interoperabilidad de servicios de E-Learning mediante procesos Web, caso de estudio Universidad del Cauca. (Tesis de pregrado, Universidad del Cauca). Popayan, Colombia.

San Cristóbal, E. (2010). Metodología, estructura y desarrollo de interfaces intermedias para la conexión de laboratorios remotos y virtuales a plataformas educativas. (Tesis doctoral, Universidad Nacional de Educación a Distancia). España.

Sotelo, F. \& Solarte, M. (2013). Revisión de tema para la incorporación de recursos web como servicios de e-learning al sistema de gestión de aprendizaje .LRN. Tecnura, 38.

Sunil, L. (2009). Integrating EduLearn Learning Content Management System (LCMS) with Cooperating Learning Object Repositories (LORs) in a Peer To Peer (P2P) architectural Framework. SICSOFT Software Engineering Notes, 34(3).

tWSDL Project. (2010). TCI WSDL Client/Server. R e cuperado de http://code.google.com/p/twsdl/

UDDI.org. (2002). UDDI Versión 2.04 API, Especificación. Recuperado de http://uddi.org/pubs/ProgrammersAPI-V2.04-Published-20020719.htm

Universidad de Valencia. (s. f.). Jabber. Recuperado de http://www.uv.es/avirtual/manual/ch08s03.html (consultado en marzo de 2013).

Universidad del Cauca. (n.d.). Comunidad virtual de la Universidad del Cauca, "Moodle". Recuperado de http://univirtual.unicauca.edu.co/moodle/

Universidad del Cauca. (n.d.). Entorno Virtual de Aprendizaje Universidad del Cauca, "EVA". Recuperado de http://eva.unicauca.edu.co

W3C. (2010). Guía Breve de Servicios Web. Recuperado de http://www.w3c.es/divulgacion/guiasbreves/ ServiciosWeb

W3C. (2007). SOAP Versión 1.2 Part 1, Recomendación. Recuperado de www.w3.org/TR/ soap12-part1/\#intro

W3C. (1999). Web Characterization Terminology \& Definitions Sheet. Recuperado de http://www. w3.org/1999/05/WCA-terms/\#Resource2

W3C. (2001). Web Services Description Language (WSDL) 1.1, Recomendación. Recuperado de www.w3.org/TR/ws-eventing

Zapata, M. (2003). Sistemas de gestión del aprendizaje - Plataformas de teleformación. Revista de Educación a Distancia, Universidad de Murcia.

\section{(C) $(1) \Theta$}


